\title{
In Memoriam Professor Ekkehard Recknagel
}

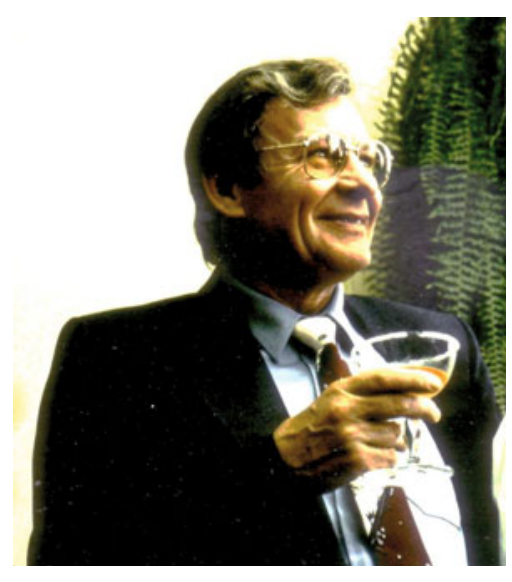

Professor Ekkehard Recknagel is widely known for his pioneering work in Nuclear Solid State Physics and Cluster Physics. He has been serving on the ISSPIC International Advisory Board since 1998; in 1990 he chaired ISSPIC 5 in Konstanz. Ekkehard died in Konstanz on June 8, 2009. He is being missed.

Ekkehard was born in Halberstadt near Göttingen on April 7, 1931. His family moved to Silesia which became part of Poland after the Second World War. The family moved back to the area of Göttingen where Ekkehard finished high school and began his studies of Physics. He transferred to the University of Heidelberg where he became a student of Hans Kopfermann, the leading expert in the analysis of hyperfine structure in atomic spectra. Ekkehard earned his Physics Diploma in 1957 with a measurement of the nuclear quadrupole moment of the Zn-67 nucleus. Three years later he received his doctoral degree for his work on hyperfine structure in Au-197 and the magnetic moment of the Au-197 nucleus.

Ekkehard's scientific career began at the University of Karlsruhe where he worked at the institute for high-frequency technology and electronics. From 1961 to 1962 he spent a post-doctoral year at Harvard University where he had the opportunity to work with Norman F. Ramsey on aspects of the hydrogen maser. Back in Germany, Ekkehard joined the scientific staff at the newly founded Hahn-Meitner Institute in Berlin where a 7 MV van de Graaff accelerator had been installed. Ekkehard's group measured magnetic dipole and electric quadrupole moments of short-lived nuclear states populated in reactions with protons or deuterons. In the course of these measurements they developed new techniques, including a stroboscopic technique that made it possible to measure the nuclear Larmor precession with enhanced sensitivity and accuracy.

The group applied these techniques to determine properties of solids, including magnetic hyperfine fields at the site of radioactive nuclei in ferromagnets, and electric field gradients arising from point defects trapped at radioactive impurity atoms in cubic crystals. This was the birth of Nuclear Solid State Physics. The power of the approach rests in its sensitivity. The required concentration of radioactive nuclei is extremely small because emitted gammas can be detected efficiently; correspondingly small levels of point defects can be detected if a significant fraction of them are trapped. Once trapped, they are "finger-printed" by the observed interaction frequency. Thermally activated diffusion of defects can then be monitored by studying the temperature dependence of the signal.

Noteworthy are the group's measurements of the Knight shift at radioactive impurities in metals. The shift in the Larmor frequency arises from the contribution of conduction electrons to the field at the nucleus. It was first reported in 1949 by Walter Knight who, together with his students, would later discover electronic shell structure in atomic clusters of simple metals. To physicists, the world may be a small place. You embark on a totally new research area, and your path may still cross the path of colleagues whom you had met in your "earlier life."

In 1975 Ekkehard was appointed Professor at the University of Konstanz. The university had been founded a few years before; the Physics Department had not yet graduated a single student. Ekkehard had three Research Associate positions to fill. Two positions went to scientists with experience in nuclear solid state physics. A $350 \mathrm{kV}$ 
ion implantator was to become the workhorse for experiments designed to study radiation damage in solids. To the laymen, Ekkehard would illustrate the technique of ion implantation with a cartoon from the "Vater \& Sohn" cartoon series by Erich Ohser. The father and his son had prepared a raisin cake to surprise their Mom, but when the cake was already in the range they realized they had forgotten to add raisins to the dough. So Dad takes his gun, loads it with raisins and implants the raisins into the cake.

Research in nuclear solid state physics was highly successful. New powerful techniques were developed or quickly adapted. Proton-induced X-ray emission (PIXE) was used for elemental analysis. Muon spin rotation experiments, conducted at the Paul-Scherrer-Institut in Switzerland, was used to study magnetic fields in solids and the diffusion of muonium, a hydrogen-like bound muon-electron pair, in semiconductors. The technique of emission channeling was developed in which charged particles, emitted upon nuclear decay, are guided by the crystalline lattice and detected outside the specimen; diffusion of defects and the nature of the lattice site occupied by the radioactive atoms could be determined.

28 students earned their doctoral degree with research in nuclear solid state physics, including three who continued toward their Habilitation and became tenured professors at universities in Germany. Ekkehard proudly published an Annual Research Report, a total of twenty until his retirement in 1996.

One of the three Research Associate positions that Ekkehard had to fill in 1975 was offered to Klaus Sattler who had excited Ekkehard with his plan to investigate atomic clusters. Ekkehard felt that techniques developed in Nuclear Solid State Physics could possibly be applied to these ultrafine particles (the word nano had not yet become fashionable).

The first goal of the cluster group was to synthesize metal clusters over a wide size range. Previous work had resulted in clusters that were either quite small, for example when sputtering was employed, or rather large when the gas aggregation technique was used, too large for the particles to be identified by mass spectrometry. It took a while because everything was built in house: several water- and liquid nitrogen cooled variants of gas aggregation sources, an ever increasing number of high temperature crucibles, a pulsed electron source for ionization, the ion optics, and the time-to-digital converter. The efforts proved successful in 1980, when Klaus Sattler, Jürgen Mühlbach and Ekkehard reported on the "Generation of Metal Clusters Containing from 2 to 500 Atoms". The report [Phys. Rev. Lett. 45, 821 (1980)] was published just before ISSPIC 2 was held in Lausanne where Klaus would proudly present mass spectra of lead, bismuth and antimony clusters.

After earning my doctoral degree in 1979 under Ekkehard's supervision for the investigation of radiation damage in copper, I had joined the cluster group. Our efforts were devoted to the generation of van der Waals clusters. An early result was the identification of "Magic Numbers for Sphere Packings - Experimental Verification in Free Xenon Clusters" published with Klaus and Ekkehard [Phys. Rev. Lett. 47, 1121 (1981)]. The interpretation in this work, namely that the observed abundance anomalies would reflect enhanced stability of neutral xenon clusters, drew quite some criticism, and was ultimately proven incorrect. Still, the idea that anomalies in mass spectra identify cluster sizes with unusual properties turned out to be quite powerful, last not least in the discovery of electronic shell structure in metal clusters, and fullerenes. Some colleagues argued that the word "magic" had no legitimate place in science but Ekkehard, with his background in nuclear physics where the term was widely used, was not bothered by that sort of criticism.

The mass spectra published in the above-mentioned reports showed an apparent abrupt loss of resolution beyond some specific cluster size; with improved resolution it became clear that the effect was due to the appearance of additional peaks with nominally half-integer cluster size, i.e. due to odd-sized doubly charged clusters. No doubly charged ions were observed below a "critical size" that ranged from 20 for NaI clusters to 52 for xenon. "Evidence for Coulomb Explosion of Doubly Charged Microclusters" was reported by Klaus, Jürgen, myself, Peter Pfau and Ekkehard in Phys. Rev. Lett. 47, 160 (1981).

Ekkehard found this topic particularly exciting because of the close analogy between fission of multiply charged clusters and fission of nuclei. The questions of fission barriers, spontaneous versus induced fission, and symmetric versus asymmetric fission play a role in both systems, in spite of the very different length scales and type of bonding. The Konstanz group established a correlation between the critical sizes of van der Waals systems and monomer size and strength of the van der Waals force; they continued to measure the size distribution of fragment ions from unimolecular fission of triply charged van der Waals clusters.

In those early years, many scientists at German universities engaged in cluster research. There was, however, no formal program through which that type of research, not to mention collaboration among researchers, could be funded. The interdisciplinary nature of the research, performed at Physics and Chemistry Departments alike, provided another barrier. Ekkehard was instrumental in convincing the DFG, the German Research Society, to provide funding through a special program named "Physics of Inorganic Clusters". Funding was also provided for collaborations and annual meetings of scientists from Germany and other German-speaking countries. Funding has long ceased but Clustertreffen are still being held each year. 


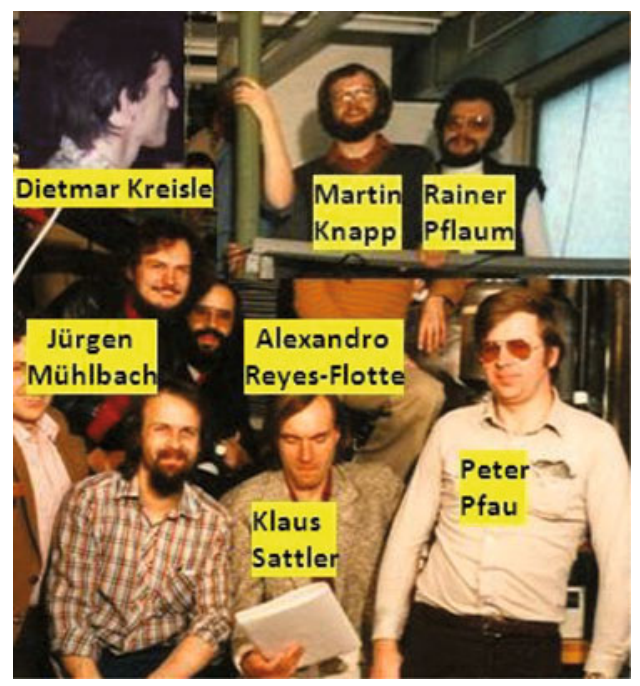

Members of the cluster group in 1983.

Two other areas of research, pursued in the 80s, should be mentioned. Negatively charged clusters were formed by attaching free electrons to pre-formed neutral clusters, including clusters of water, ammonia, and carbon dioxide (M. Knapp, O. Echt, D. Kreisle, E. Recknagel, J. Phys. Chem. 91, 2601 (1987)). Another topic of interest were magic numbers in alkali halide clusters. Depending on the way the clusters were ionized, one could either observe anomalies related to particularly stable cluster ions, or neutrals (R. Pflaum, K. Sattler, E. Recknagel, Chem. Phys. Lett. 138, 8 (1987)). Over the years, 14 students received a doctoral degree for their work in cluster science.

In 1987 Klaus Sattler accepted a professorship at the University of Hawaii; I left Konstanz shortly thereafter. The cluster group continued their work until Ekkehard's retirement; Dietmar Kreisle led the cluster group during those years. Projects included the characterization of thermionic emission from hot clusters of refractory metals, measurement of the kinetic energy distribution of the electrons, and photon emission in the visible and near-UV. It should be mentioned that several of the projects mentioned above were done in collaboration with other research groups, in particular with Tilmann Märk's group at the University of Innsbruck, Gerd Gantefoer, Wolfgang Eberhard and others at the Research Center Jülich, and Lutz Schweikhard and co-workers at the University of Greifswald.

In 1986 research in Ekkehard's group was abruptly brought to a stand still. On April 26, the melt down of one of the reactors at the nuclear power plant in Chernobyl/Ukraine released a huge amount of radioactive material into the environment. The public didn't learn of the disaster until April 28 when the radioactive cloud had reached Sweden. Two days later, a heavy rain storm precipitated radioactive isotopes, in particular short-lived iodine-131 and long-lived cesium-137, over some areas in South Germany. Ekkehard's group was prepared to make use of their instruments and expertise. Over the next few weeks they systematically analyzed rainwater, soil, fresh produce from the farms, grass from the meadows, and diary products. They analyzed all kinds of samples that concerned local residents would bring to the institute, including carcasses of wildlife. On some days one had to hold ones breath when entering the institute. High levels of contamination were found in many samples. The public was informed through newspapers, radio, TV, and oral presentations in the communities; the results were later compiled in a book (G. Lindner and E. Recknagel, Tschernobyl-Auswirkungen auf die Bodensee Region. Thorbecke, Sigmaringen, 1988). Parents were advised to keep their children from playing in sandboxes; farmers were advised to keep their cows in the sheds and to mow and destroy the grass on their meadows. These warnings were not welcome by some politicians who tried to downplay the risks. Some would even blame Ekkehard for fear-mongering but ultimately the advise and authority of Ekkehard's group was widely accepted. The degree of contamination varied wildly from one location to another, it just happened to be particularly strong in the Konstanz area.

In September 1990 ISSPIC 5 was held in Konstanz. The conference was originally slated to be held in the UK, but one year before the event the group in the UK decided that they could not possibly do it. Ekkehard reluctantly agreed to organize ISSPIC 5 on short notice even though he was scheduled to undergo major surgery for cancer in early 1990. There were several hick-ups during the preparation but somehow, miraculously, all the pieces fell into place at the last minute. The symposium featured 33 invited talks and 295 contributed papers; it was attended by 400 participants.

The conference was held two weeks before the journal Nature would publish the report by Kraetschmer, Huffman and coworkers on the synthesis of $\mathrm{C}_{60}$ in macroscopic amounts [Nature 347, 354 (1990)]. These news had not yet made it to Konstanz; not a single invited contribution or Hot Topic was devoted to fullerenes. Rick Smalley had been invited but his talk was supposed to be on metal cluster chemistry. Wolfgang Kraetschmer accompanied Rick Smalley to Konstanz; on the eve of the conference we learnt from them of the news. We had difficulties finding a time slot for an additional Hot Topic presentation, but Rick Smalley graciously donated some of his time to let Wolfgang Kraetschmer 
report on the discovery of $\mathrm{C}_{60}$ crystals. Ultimately the cover page of the proceedings was graced with an image of $\mathrm{C}_{60}$, and Kraetschmer contributed an article on "How we came to produce $\mathrm{C}_{60}$-fullerite" (Z. Phys. D 19, 405 (1991)).

The world may appear small to physicists, but not to Ekkehard who had a wide range of interests. He devoured books, especially non-fiction books including books on history, culture, biographies, books on the chemistry of preparing food, to name a few. He would visit historic sites and exhibits of modern arts. There was hardly any topic that would not capture his interest and engagement. He was tolerant but he had reasoned positions on many issues. He was highly critical of religion, and even more critical of the role of the church in society. He was highly critical of claims that were in conflict with the fundamental laws of physics, as we know them. He remained skeptic of the promises of wholesome nutrition. Claims of the healing power of highly diluted medicine, as routinely prescribed by homeopaths, would infuriate him. They use dilutions that would leave less than one molecule in all the water that is in the Lake of Constance, he would exclaim in disgust.

Ekkehard was an imminently social person. His hospitality was outstanding. Once a year his group would celebrate the beginning of summer. These parties would often continue into the early morning hours. All were invited, including the families of everyone with ties to the institute, from the mechanics working in the machine shop to the janitor. Ekkehard would be seen conversing with them; discussion of research was taboo during those parties. Ekkehard felt responsible for training his students and for providing an environment in which students and staff scientists could thrive, but he was also deeply concerned about the well-being of students, scientific and non-scientific staff at his institute, including their families.

No efforts were spared to celebrate major accomplishments. When a doctoral student graduated the institute would spend the rest of the day celebrating. Ekkehard would open the event by reading a detailed laudation, written in prose and presented in an elaborate way. Major events like a habilitation (which, in Germany, qualifies a scientist to apply for a tenured professorship) were usually celebrated with a procession through the City of Konstanz. Of course, Ekkehard would also enjoy being at the focus of a celebration meticulously organized by members of his institute, for example on the occasion of his 60th birthday, and his retirement.

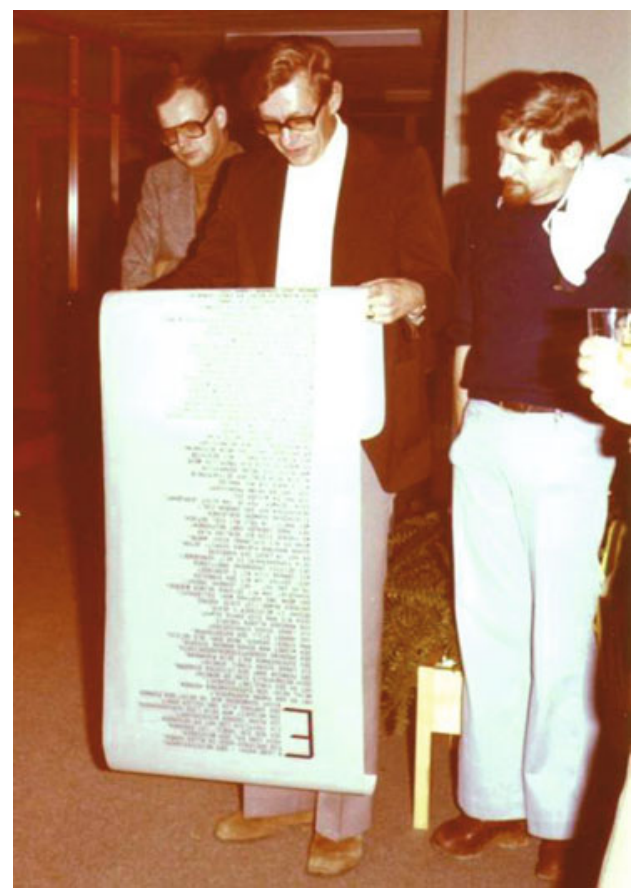

Ekkehard in 1976, presenting a laudation written in prose.

Ekkehard had suffered from cancer during the last two decades but he remained optimistic; he would always maintain an upbeat attitude. Still, he also was a realist. Eventually he came to realize that there was no cure. He had consulted with several medical cancer experts, and ultimately accepted the fact that there was nothing they could do. He would refuse to seek help from non-traditional treatments; instead he sought to make the best of the time that was left to him. During the last few months he was in pain, but he would still enjoy visits by his former students, his friends, his family.

Ekkehard died at his home in Konstanz on June 8 2009. He will be remembered for his scientific accomplishments and his human warmth. 